OPEN ACCESS

\section{Performance of upstream interaction region detectors for the FIRST experiment at GSI}

To cite this article: Z Abou-Haidar et al 2012 JINST 7 P02006

View the article online for updates and enhancements.

\section{Related content}

\begin{abstract}
FIRST experiment: Fragmentation of lons Relevant for Space and Therapy C Agodi, Z Abou-Haidar, M A G Alvarez et al.

- Precise measurement of prompt photon emission from $80 \mathrm{MeV} / \mathrm{u}$ carbon ion beam irradiation

C Agodi, F Bellini, G A P Cirrone et al.
\end{abstract}

Performance of the LHCb muon system with cosmic rays

M Anelli, R Antunes Nobrega, G

Auriemma et al.

\section{Recent citations}

$$
\begin{aligned}
& \text { - Secondary radiation measurements for } \\
& \text { particle therapy applications: Charged } \\
& \text { secondaries produced by } 160 \text { ion beams } \\
& \hline \text { in a PMMA target at large angles } \\
& \text { A. Rucinski et al } \\
& \text { - Secondary radiation measurements for } \\
& \text { particle therapy applications: charged } \\
& \text { particles produced by }{ }^{4} \mathrm{He} \text { and }{ }^{12} \mathrm{C} \text { ion } \\
& \text { beams in a PMMA target at large angle } \\
& \text { A Rucinski et al } \\
& \text { - Secondary radiation measurements for } \\
& \text { particle therapy applications: prompt } \\
& \text { photons produced by }{ }^{4}{ }^{1}{ }^{12} \mathrm{C} \text { and }{ }^{16} \mathrm{O} \\
& \frac{\text { ion beams in a PMMA target }}{\text { I Mattei et al }}
\end{aligned}
$$




\section{Performance of upstream interaction region detectors for the FIRST experiment at GSI}

Z. Abou-Haidar, ${ }^{m}$ C. Agodi, ${ }^{d}$ M.A.G. Alvarez, ${ }^{m}$ M. Anelli, ${ }^{a}$ T. Aumann, ${ }^{v}$ G. Battistoni, ${ }^{a}$ A. Bocci, ${ }^{m}$ T.T. Böhlen, ${ }^{r, s}$ A. Boudard, ${ }^{q}$ A. Brunetti, ${ }^{b, k}$ M. Carpinelli, ${ }^{b, k}$

G.A.P. Cirrone,${ }^{d}$ M.A. Cortes-Giraldo,${ }^{x}$ G. Cuttone,${ }^{d}$ M. De Napoli, ${ }^{d}$ M. Durante,${ }^{v}$ J.P. Fernández-García, ${ }^{x}$ C. Finck, ${ }^{p}$ M. I. Gallardo, ${ }^{x}$ B. Golosio, ${ }^{b, k}$ E. larocci, ${ }^{c, h}$ F. lazzi, ${ }^{f, i}$ G. Ickert, ${ }^{v}$ R. Introzzi, ${ }^{f}$ D. Juliani, ${ }^{p}$ J. Krimmer, ${ }^{y}$ N. Kurz, ${ }^{,}$M. Labalme, ${ }^{o}$ Y. Leifels, ${ }^{v}$ A. Le Fèvre, ${ }^{v}$ S. Leray, ${ }^{q}$ F. Marchetto, ${ }^{f}$ V. Monaco, ${ }^{f, w}$ M.C. Morone,,${ }^{g, z}$ P. Oliva, ${ }^{b, k}$ A. Paoloni, ${ }^{c}$ V. Patera, ${ }^{a, b}$ L. Piersanti, ${ }^{c, h}$ R. Pleskac, ${ }^{\nu}$ J.M. Quesada, ${ }^{x}$ N. Randazzo, ${ }^{j}$ F. Romano, ${ }^{d, u}$ D. Rossi, ${ }^{v}$ V. Rosso, ${ }^{l, n}$ M. Rousseau, ${ }^{p}$ R. Sacchi, ${ }^{f, w}$ P. Sala, ${ }^{a}$ A. Sarti, ${ }^{c, h, 1}$ C. Schuy, ${ }^{\nu}$ A. Sciubba, ${ }^{c, h}$ C. Sfienti, ${ }^{d, s}$ H. Simon, ${ }^{\nu}$ V. Sipala,,${ }^{j, k}$ E. Spiriti, ${ }^{e}$ L. Stuttge, ${ }^{p}$ S. Tropea ${ }^{d}$ and H. Younis ${ }^{f, i}$

\footnotetext{
${ }^{a}$ Istituto Nazionale di Fisica Nucleare - Sezione di Milano, Milano, Italy

${ }^{b}$ Istituto Nazionale di Fisica Nucleare - Sezione di Cagliari, Cagliari, Italy

${ }^{c}$ Istituto Nazionale di Fisica Nucleare - Laboratori Nazionali di Frascati, Frascati, Italy

${ }^{d}$ Istituto Nazionale di Fisica Nucleare - Laboratori Nazionali del Sud, Catania, Italy

${ }^{e}$ Istituto Nazionale di Fisica Nucleare - Sezione di Roma 3, Roma, Italy

${ }^{f}$ Istituto Nazionale di Fisica Nucleare - Sezione di Torino, Torino, Italy

${ }^{g}$ Istituto Nazionale di Fisica Nucleare - Sezione di Roma Tor Vergata, Roma, Italy

${ }^{h}$ Dipartimento di Scienze di Base e Applicate per l'Ingegneria, “La Sapienza” Università di Roma, Roma, Italy

${ }^{i}$ Dipartimento di Fisica, Politecnico di Torino, Torino, Italy

${ }^{j}$ Istituto Nazionale di Fisica Nucleare - Sezione di Catania, Catania, Italy

${ }^{k}$ Università di Sassari, Sassari, Italy

${ }^{l}$ Istituto Nazionale di Fisica Nucleare - Sezione di Pisa, Pisa, Italy

${ }^{m}$ CNA, Sevilla, Spain

${ }^{n}$ Università di Pisa, Pisa, Italy

${ }^{o}$ LPC-Caen, ENSICAEN, Universite de Caen, CNRS/IN2P3, Caen, France

${ }^{p}$ Institut Pluridisciplinaire Hubert Curien, Strasbourg, France

${ }^{q}$ CEA-Saclay, IRFU/SPhN, Gif sur Yvette Cedex, France
}

\footnotetext{
${ }^{1}$ Corresponding author.
} 
${ }^{r}$ European Organization for Nuclear Research CERN, Geneva, Switzerland

${ }^{s}$ Medical Radiation Physics, Karolinska Institutet and Stockholm University, Stockholm, Sweden

${ }^{t}$ Universitat Mainz Johann-Joachim-Becher, Mainz, Germany

"Centro Studi e Ricerche e Museo Storico della Fisica "Enrico Fermi", Roma, Italy

${ }^{v}$ GSI Helmholtzzentrum fur Schwerionenforschung, Darmstadt, Germany

${ }^{w}$ Dipartimento di Fisica, Università di Torino, Torino, Italy

${ }^{x}$ Departamento de Fisica Atomica, Molecular y Nuclear, University of Sevilla, 41080-Sevilla, Spain

${ }^{y} I P N-L y o n$, Universite de Lyon, Universite Lyon 1, CNRS/IN2P3, Villeurbanne, France

${ }^{z}$ Dipartimento di Biopatologia e Diagnostica per Immagini, Università di Roma Tor Vergata, Roma, Italy

E-mail: Alessio.Sarti@uniroma1.it

ABSTRACT: The FIRST (Fragmentation of Ions Relevant for Space and Therapy) experiment at GSI has been designed to study carbon fragmentation, measuring ${ }^{12} \mathrm{C}$ double differential cross sections $\left(\partial^{2} \sigma / \partial \theta \partial E\right)$ for different beam energies between 100 and $1000 \mathrm{MeV} / \mathrm{u}$. The experimental setup integrates newly designed detectors in the, so called, Interaction Region around the graphite target. The Interaction Region upstream detectors are a $250 \mu \mathrm{m}$ thick scintillator and a drift chamber optimized for a precise measurement of the ions interaction time and position on the target. In this article we review the design of the upstream detectors along with the preliminary results of the data taking performed on August 2011 with $400 \mathrm{MeV} / \mathrm{u}$ fully stripped carbon ion beam at GSI. Detectors performances will be reviewed and compared to those obtained during preliminary tests, performed with $500 \mathrm{MeV}$ electrons (at the BTF facility in the INFN Frascati Laboratories) and $80 \mathrm{MeV} / \mathrm{u}$ protons and carbon ions (at the INFN LNS Laboratories in Catania).

KEYWORDS: Wire chambers(MWPC, Thin-gap chambers, drift chambers, drift tubes, proportional chambers etc); Gaseous detectors; Scintillators, scintillation and light emission processes (solid, gas and liquid scintillators); Particle tracking detectors (Gaseous detectors) 


\section{Contents}

1 Introduction 1

2 The experimental setup 2

2.1 The Beam Test Facility (BTF) 2

2.2 The LNS zero degree test setup 2

2.3 DAQ system 2

3 The Start Counter 3

3.1 Start Counter performances: efficiency and time resolution 4

4 The Beam Monitor drift chamber 6

4.1 Track reconstruction 7

4.2 Hit detection efficiency 8

4.3 Spatial resolution 9

5 Detector performance in the FIRST experiment at GSI 11

$\begin{array}{lll}5.1 & \text { Start Counter preliminary results } & 11\end{array}$

5.2 Beam Monitor preliminary results 12

6 Conclusions $\quad 14$

\section{Introduction}

The FIRST (Fragmentation of Ions Relevant for Space and Therapy) experiment [1] is performed at the GSI heavy ion synchrotron (SIS) in Darmstadt. Its aim is to measure carbon fragmentation on nuclei at different energies between 100 and $1000 \mathrm{MeV} / \mathrm{u}$. The study of fragmentation processes is relevant to different fields of physics, including both basic research and applications. In particular, different reaction mechanisms can be better understood by means of the analysis of double differential cross sections as a function of the energy and of the angle with respect to the beam.

The FIRST experimental setup consists of two main regions: the Magnet Region and the Interaction Region. While the former integrates an already existing setup composed by a dipole magnet (ALADiN, [2]), a TPC (Music IV, [3]), a neutron detector (LAND, [4]) and a time of flight scintillator wall, the latter is made up of newly designed detectors installed around the target.

The FIRST setup aims for a suitable particle identification capability providing a $\frac{\sigma_{M}}{M} \leq 10 \%$, (where $\mathrm{M}$ is the fragment mass). A $10 \%$ relative uncertainty on the fragment mass is mandatory in order to have a clear separation of all the ions and isotopes under study. The required precision on fragment mass directly translates into requirements on the time and momentum resolution for all the detectors used in the FIRST experiment. The mass measured in the spectrometer is given 
by $M=k|R| f(\beta)$ where $k=\frac{0.3 Z}{m_{0}}$ and $f(\beta)=\frac{\sqrt{1-\beta^{2}}}{\beta}=\frac{1}{\beta \gamma}$. The relative uncertainty on M is hence related to the time and momentum resolutions by the relation

$$
\left(\frac{\sigma_{M}}{M}\right)^{2}=\left(\frac{\sigma_{p}}{p}\right)^{2}+\left(\gamma \frac{\sigma_{t}}{t}\right)^{2}
$$

where $\frac{\sigma_{p}}{p}=\frac{\sigma_{R}}{R}$ has been used.

The Interaction Region target upstream detectors are a thin scintillation counter (Start Counter), designed for triggering and timing purposes, a drift chamber (Beam Monitor) dedicated to a precise ion impinging point measurement and a pre-target fragmentation detection, while the downstream ones are a silicon Vertex Detector and a thick scintillator (Proton Tagger) used to detect light fragments produced at large angles.

In this paper we review the design of the upstream detectors as well as test beam results obtained with $500 \mathrm{MeV}$ electrons (at the Beam Test Facility of the INFN LNF Laboratories in Frascati) and with $80 \mathrm{MeV} / \mathrm{u}$ protons and carbon ions (at the Zero Degree Line of the INFN LNS Laboratories in Catania). Section 2 describes the experimental setup, the test beam facilities and the DAQ for the two tests that have been performed. The Start Counter is described in section 3, the Beam Monitor in section 4. The preliminary results of the FIRST experiment obtained at GSI are shown in section 5 .

\section{The experimental setup}

\subsection{The Beam Test Facility (BTF)}

The drift chamber has been tested at the BTF experimental facility (figure 1), with $500 \mathrm{MeV}$ electrons extracted in $10 \mathrm{~ns}$ spills at a frequency of $25 \mathrm{~Hz}$. In each spill the number of electrons followed a Poisson distribution with $\overline{N_{e}}=1$. A lead glass calorimeter (Beam Dump) was used to select single electron events. An example of the calorimeter charge distribution measured during the spills is shown in figure 2. The RF signal from the accelerator has been used to trigger the acquisition.

\subsection{The LNS zero degree test setup}

The experimental setup at the LNS zero degree line is shown in figure $3.80 \mathrm{MeV} / \mathrm{u}$ protons and fully stripped ${ }^{12} \mathrm{C}$ ions were delivered at $\sim 1 \mathrm{MHz}$, in order to reproduce GSI-like conditions. A $4 \times 4 \times 8 \mathrm{~cm}^{3}$ plastic scintillator (BC-404) was used as beam dump as well as a trigger. The event rate was $\sim 1 \mathrm{kHz}$, due to the electronics dead time.

\subsection{DAQ system}

Both tests on beam shared the same NIM and VME electronics. While the former is used for the trigger logic and handles the dead time of the DAQ, the latter comprises a 64 channels multihitmultievent CAEN V1190B TDC (with 100 ps time resolution and $1 \mu$ s window width) and a 16 channels CAEN V792N QDC (with $0.1 \mathrm{pC}$ resolution over a charge conversion window ranging from 0 to $400 \mathrm{pC}$ ). Data acquisition is performed by means of a Motorola MVME5100 CPU. The low voltage for the electronics of the Start Counter and the Beam Monitor is supplied by a low ripple low noise quad independent DC channel GW INSTEK GPS4303, while a CAEN N1470 


\section{Beam Dump}

\section{Drift Chamber}

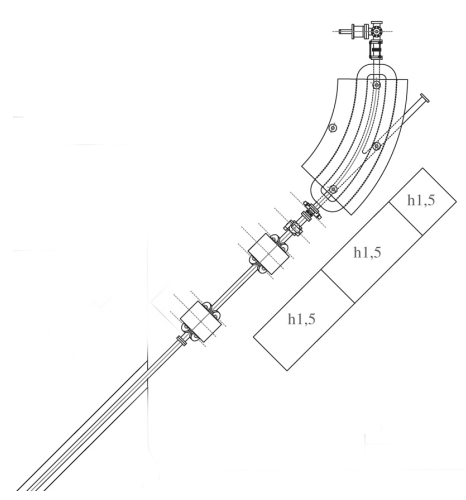

Figure 1. Experimental setup at the BTF facility; the position of the various detectors is shown, as well as the other elements of the setup.

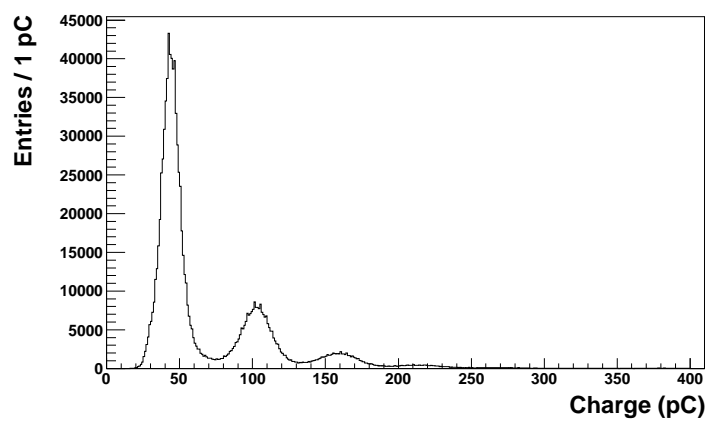

Figure 2. Typical BTF beam dump calorimeter charge histogram. The various peaks shown in the histogram are related to $0,1, \ldots, \mathrm{n}$ detected particles.

four channels programmable HV power supply provides Beam Monitor high voltage. Every signal coming from the Start Counter has been split in order to measure both time and charge. Signals coming from the Beam Monitor are discriminated and then read by a TDC. Trigger time signal is also acquired in order to fix a time reference. During tests, temperature and pressure have been recorded by a barometric weather station.

\section{The Start Counter}

The Start Counter has been designed for triggering and timing purposes. It has been optimized through a careful balancing of detector time resolution (time of flight measurements will be used to discriminate the different fragments) and thickness minimization. The design goal was to have a pre-target particle interaction probability of less than $1 \%$ with respect to the on-target one, reducing to a negligible amount the Start Counter contribution to the cross section measurement systematics. 


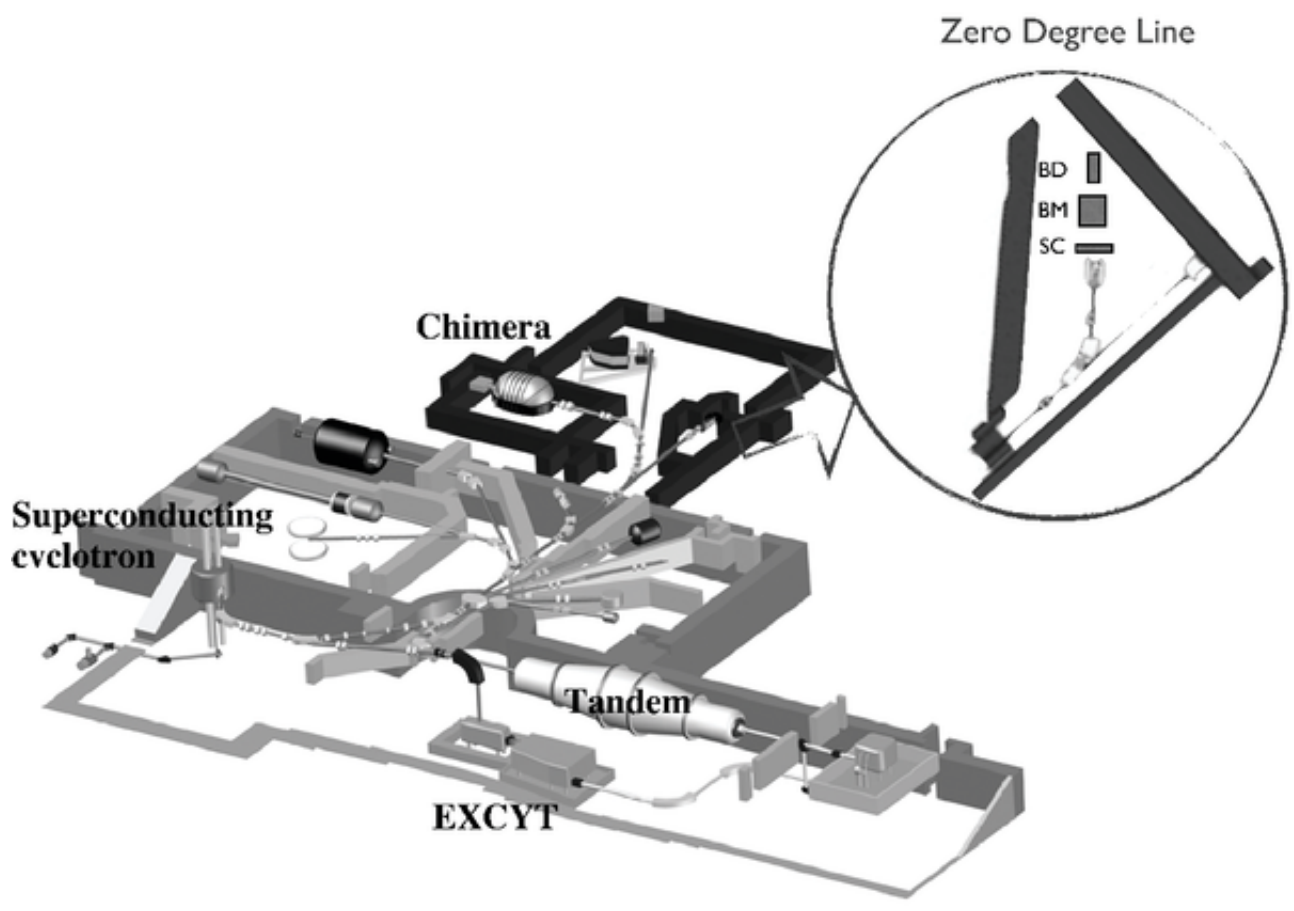

Figure 3. Radioactive ion beam facility at INFN LNS. The experimental setup at the Zero Degree Line is shown as well as the other elements of the setup.

In FIRST a 5-8 mm thick graphite target was foreseen, so the final Start Counter layout, taking into account the different material density and interaction cross sections, was designed as a $250 \mu \mathrm{m}$ thick disc, $52 \mathrm{~mm}$ diameter, of plastic scintillator (EJ-228). Light is collected by means of 160 , $1 \mathrm{~mm}$ diameter, step-index plastic optical fibers (reference standard: IEC 60793-2-40) radially glued and grouped in four bundles, and finally connected to four fast Hamamatsu UBA H10721201 photomultipliers (40\% quantum efficiency).

The fibers have a low attenuation $(<200 \mathrm{~dB} / \mathrm{km})$ for the scintillator output wavelength $(400 \mathrm{~nm})$ and their layout has been chosen in order to maximize the light collection from the scintillator. A picture of the Start Counter is shown in figure 4.

A temporal resolution of the order of $250 \mathrm{ps}$ (standard deviation) must be achieved, in order to fulfill the precision on fragment time of flight measurement required by FIRST. The signals coming from the four photomultipliers are amplified by a factor of twenty by means of custom electronics boards, designed by the LNF electronics workshop and embedded in the detector. During LNS test beam the Start Counter has been tested, scanning different thresholds and different PMT gain values, in order to find the most suitable working point.

\subsection{Start Counter performances: efficiency and time resolution}

Two main parameters have been chosen to benchmark the Start Counter: efficiency and time resolution. The efficiency has been defined as the fraction of passing particles that fire at least 3 PMTs out of 4 . 


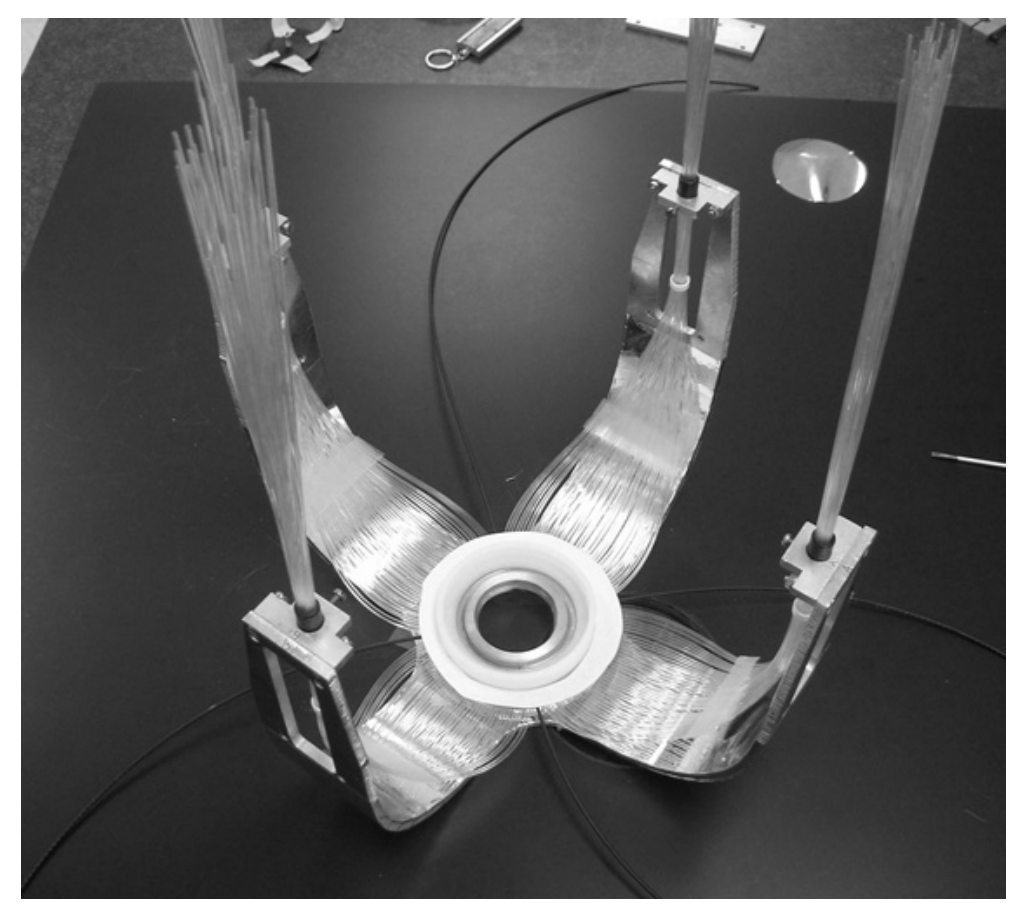

Figure 4. Start Counter assembly.

PMT time and charge information have been acquired in order to carry out time slewing correction on carbon ions, so as to refine and adjust the final results.

The charge-time correlation distributions that have been used to compute the correction are shown in figure 5 , choosing as fitting function:

$$
T=p_{0}+\frac{p_{2}}{\left(Q-p_{1}\right)^{2}} .
$$

No time slewing correction has been applied on proton data, since their measured charge variation was not appreciable.

To estimate the time resolution, after equalization of the delays of the four TDC channels, we performed a Gaussian fit to the time difference distribution of two Start Counter readout photomultipliers, as shown in figure 6. All the possible combinations of photomultipliers were used to measure the time resolutions, giving results consistent within $10 \mathrm{ps}$.

Using the data sample taken at LNS, both efficiency and time resolution have been evaluated as functions of the discrimination threshold with protons ( $\sim 1 \mathrm{M}$ events) and with carbon ions ( $\sim 1.5 \mathrm{M}$ events). The results are shown in figure 7 .

Since the energy release of ${ }^{12} \mathrm{C}$ in the FIRST experiment is comprised between that of protons (lower limit) and of $80 \mathrm{MeV} / \mathrm{u}$ carbons (upper limit) available at LNS, the results obtained with $\mathrm{p}$ and ${ }^{12} \mathrm{C}$ must be interpreted as worst and best-case scenarios, respectively, for assessing detector performances. For this reason the efficiency evaluated with carbon ions is very promising, showing a flat distribution consistent with $100 \%$ in the full threshold range, while the results obtained with protons are useful to constrain detector limitations. 


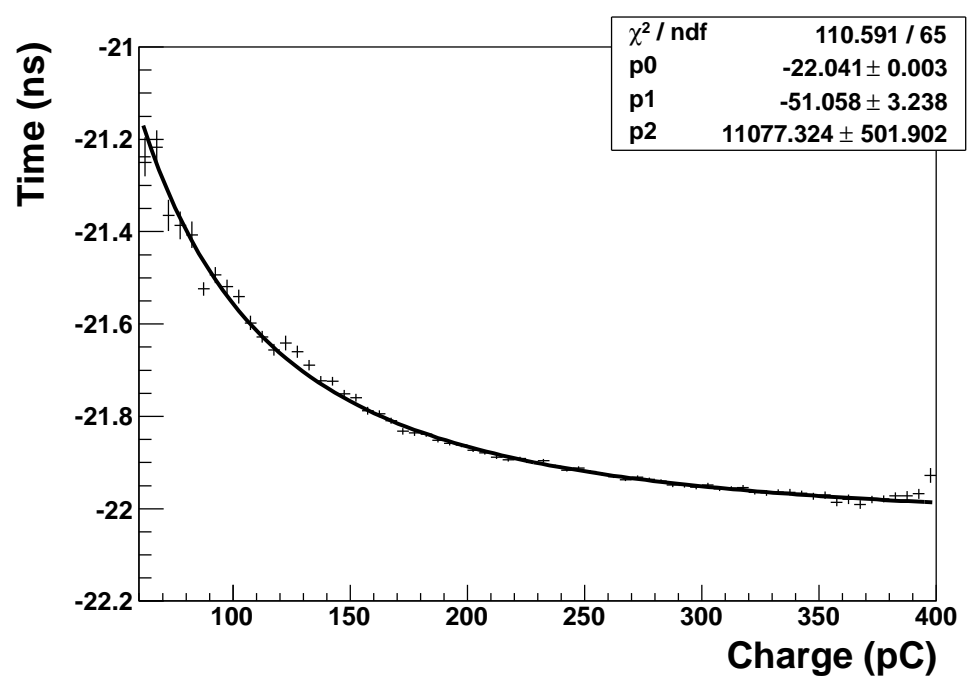

Figure 5. Charge-time correlation distribution of a Start Counter PMT.

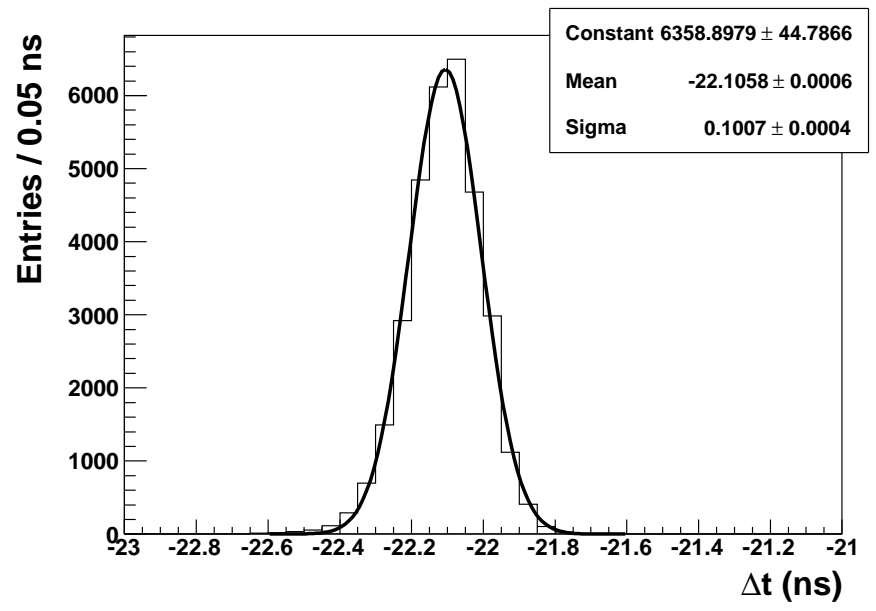

Figure 6. Time difference $(\Delta t)$ distribution of two Start Counter readout photomultipliers, obtained on LNS data (Carbon ions). A fit with a Gaussian function is superimposed.

\section{The Beam Monitor drift chamber}

The Beam Monitor, shown in figure 8, is a drift chamber designed for charged particles trajectory reconstruction. This detector provides the ion impinging point on the target, together with valuable information on possible projectile pre-target fragmentation. The requirements for fragmentation measurements in FIRST are a single cell spatial resolution $<200 \mu \mathrm{m}$ with an high particle detection efficiency. The detector is made of alternated horizontal and vertical wire layers (or planes). Each layer is composed of three rectangular cells, $16 \mathrm{~mm} \times 10 \mathrm{~mm}$ along the beam direction, for a total of 36 sense wires (see figure 9). The geometrical layout has been optimized in order to minimize ions interactions with the wires still maintaining the required cell resolution. The twelve planes (six on each "view") provide tracking redundancy and ensure a high tracking efficiency and an excellent spatial resolution. In order to minimize tracking ambiguities, the consecutive layers of each view 

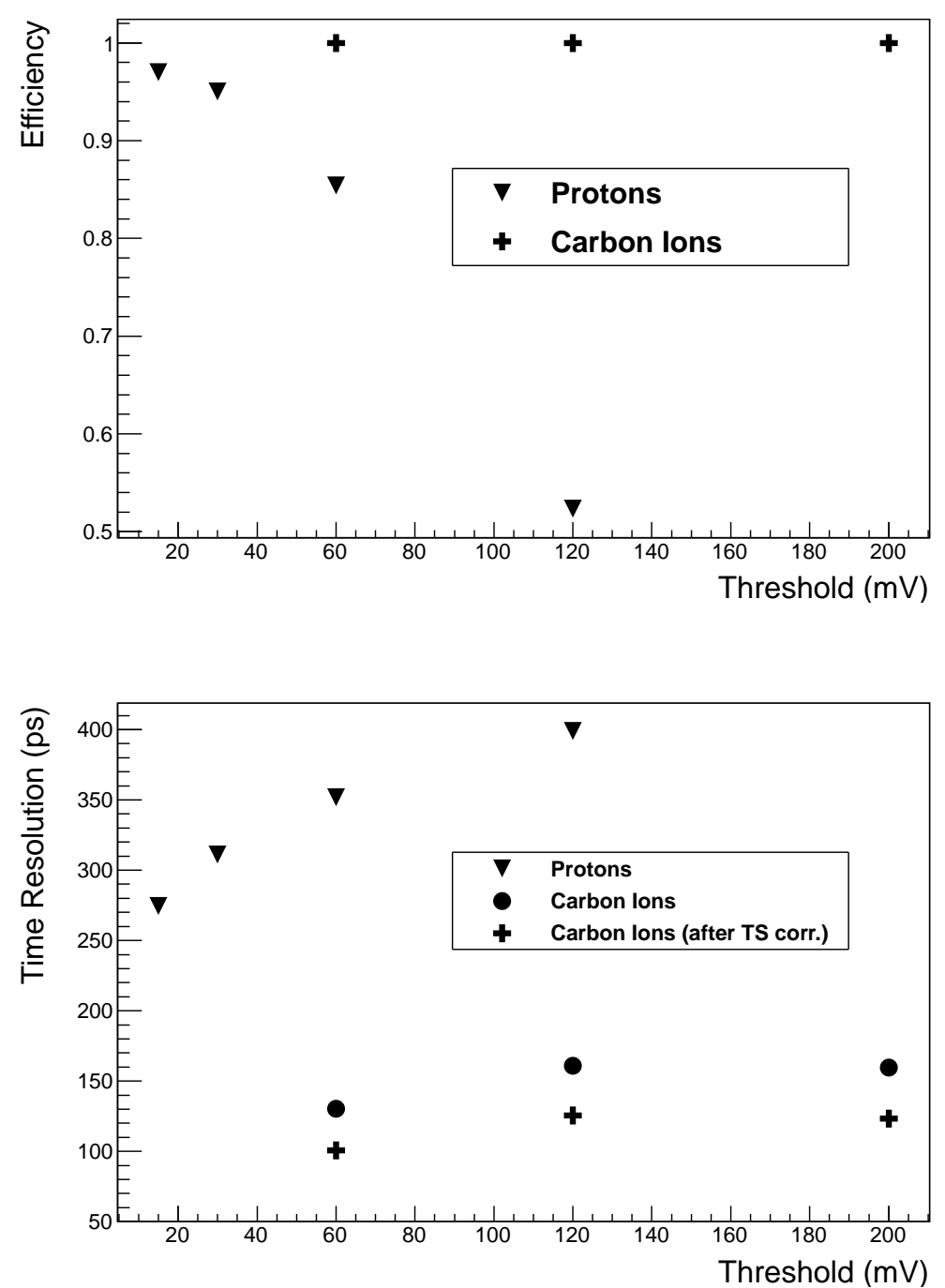

Figure 7. Start Counter performances measured at LNS: the top picture shows the efficiency versus threshold for protons (full triangle) and carbon ions (full cross); the bottom picture shows the time resolution versus threshold for protons (full triangle), carbon ions with (full cross) and without (full circle) time slewing correction. Error bars (statistical uncertainty) are smaller than marker size.

are staggered by half a cell. Custom front-end electronics boards, designed by the LNF electronics workshop, are embedded in the detector and provide wire signal amplification by a factor of 10 . Different gas mixtures, as well as operating high voltages, have been tested on electron, proton and carbon ion beams in order to choose the most suitable operating point. Hydrocarbons free mixtures are preferred because of safety issues.

\subsection{Track reconstruction}

The hits recorded by the Beam Monitor are used to reconstruct the trajectories of charged particles in the detector active volume; furthermore, such information is fundamental to discriminate 


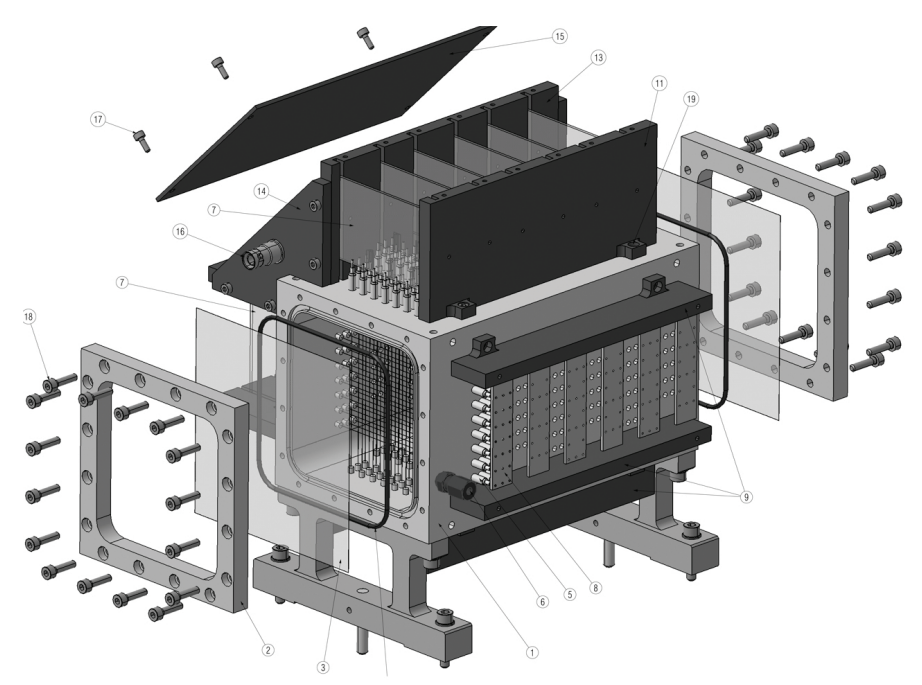

Figure 8. Beam Monitor mechanical drawing.

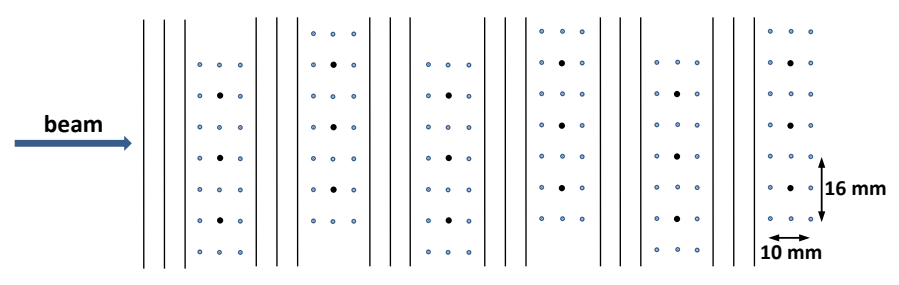

Figure 9. Beam Monitor cells layout.

between single and multiple particle events. For this purpose, a custom tracking algorithm, based on a simple least squares iterative fitting method (designed by P. Avery [5]), has been developed.

The temporal information of each hit, measured by the TDC, is converted into the distance from the fired sense wire using the space-time (s-t) curves, that in our post-processing routine are obtained with a data-based iterative procedure. In this way, we properly take into account the corrections to the initial assumption of a constant drift velocity (used as seed for the first iteration step). An example of s-t and velocity-time (v-t) relations, obtained at the end of the iterative procedure, for the Beam Monitor chamber operated at $1.8 \mathrm{kV}$ with a gas mixture of $\mathrm{Ar} / \mathrm{CO}_{2} 80 \% / 20 \%$, is shown in figure 10 .

The spatial information extracted from s-t relations is then employed in a pattern recognition routine that selects track candidates to be fitted. Only "clean" events are used for this calibration, requiring at least three single hits on each view, and using only single hits to build the tracks. A single hit occurs when only one cell per plane is fired.

\subsection{Hit detection efficiency}

The chamber performances have been evaluated in terms of efficiency and spatial resolution. Datasets were available for $500 \mathrm{MeV}$ electrons ( $\sim 7 \mathrm{M}$ events) and for $80 \mathrm{MeV} / \mathrm{u}$ protons and carbon ions ( $\sim$ 6.5 M events); different gas mixtures $\left(\mathrm{Ar} / \mathrm{CO}_{2} 80 \% / 20 \%, \mathrm{Ar} / \mathrm{CO}_{2} 90 \% / 10 \%, \mathrm{P} 10\right)$ and operating 

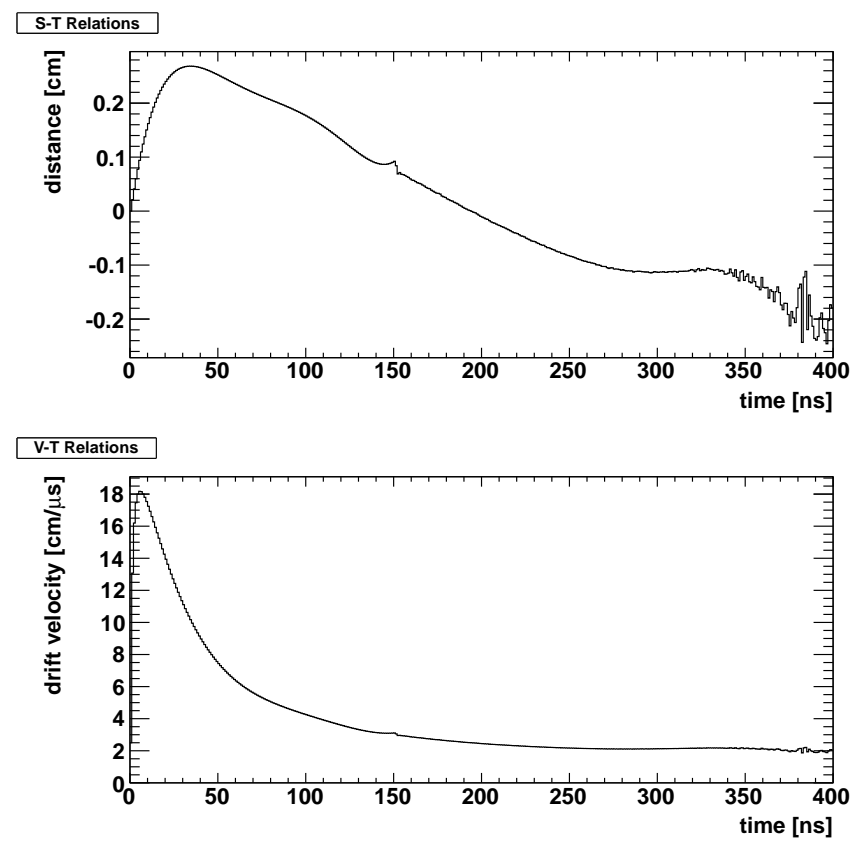

Figure 10. Space (distance from wire) — time and velocity — time relations obtained operating the Beam Monitor at $1.8 \mathrm{kV}$ with an $\mathrm{Ar} / \mathrm{CO}_{2} 80 \% / 20 \%$ gas mixture. The small bump at $150 \mathrm{~ns}$ is induced by our mathematical description of the s-t relations, since it uses different parametrizations in the 0 to 150 and 150 to 400 ns ranges.

high voltages have been tested. In the analysis of the BTF data, the Beam Dump was used to reject events with more than one electron.

Two different algorithms have been used to define the efficiency. The first, referred to as raw efficiency, has been introduced in order to find a proper working point of the detector. In fact, raw efficiency is evaluated, for each plane, as the fraction of events that have at least one hit detected on even (odd) planes, when three single hits on odd (even) planes have been scored. Hence, this is a hardware-based definition that does not account for any tracking information. Raw efficiency results, averaged over the two views and then over the entire chamber, are shown in figure 11.

In order to assess the single cell efficiency with respect to the particle distance from the wires, a second algorithm, based on the tracking method described in section 4.1, has been defined. The reconstructed tracks are used to compute the efficiency as a function of the closest distance of the charged particles from the sense wires. We found that the efficiency does not depend on the distance showing a flat behaviour around the mean values as reported in figure 11 .

\subsection{Spatial resolution}

The drift chamber spatial resolution has been evaluated by means of the tracking algorithm described in section 4.1, using the tracks reconstructed from the hits detected in the beam monitor. For each hit in each plane we compute the difference ( $\Delta x$, referred to as residual in the following) between the measured position $\left(x_{\text {meas }}\right)$ and the position of the track $\left(x_{\text {track }}\right)$. The distribution of $\Delta x$ is used to evaluate the spatial resolution of the detector. An example of the residuals distribution, fitted with a double Gaussian function, obtained for the Beam Monitor operated at $1.8 \mathrm{kV}$ with a 

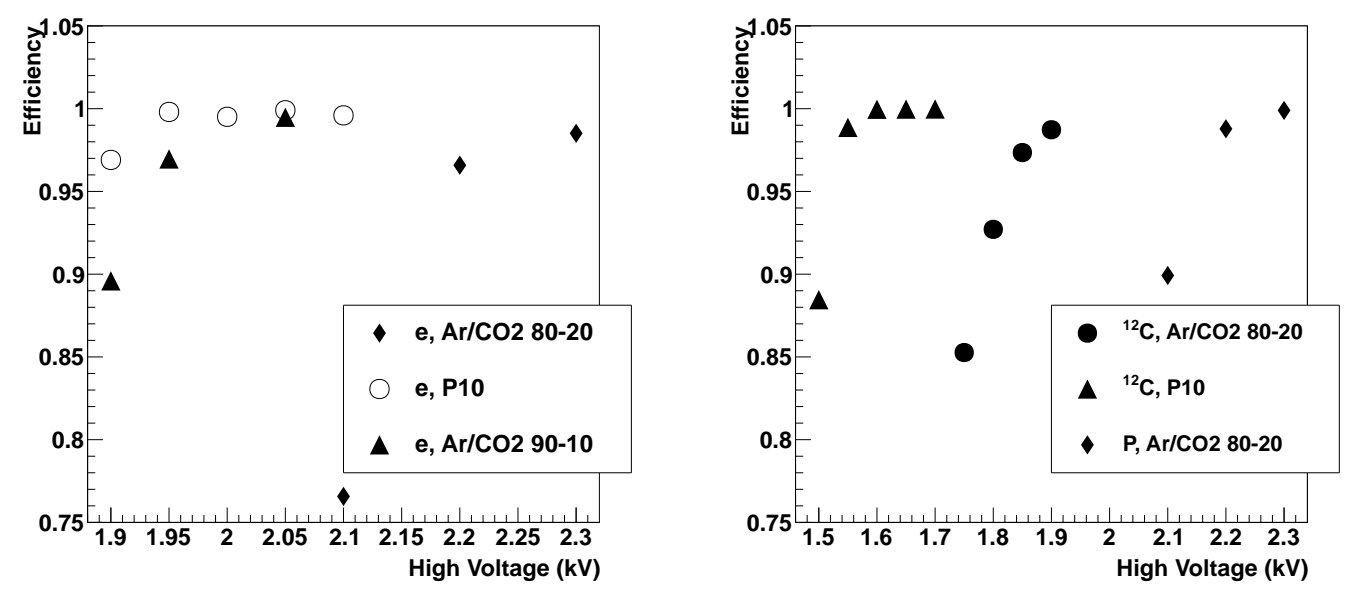

Figure 11. Beam Monitor raw efficiency results obtained at BTF (left) and LNS (right) with different gas mixtures and high voltages.

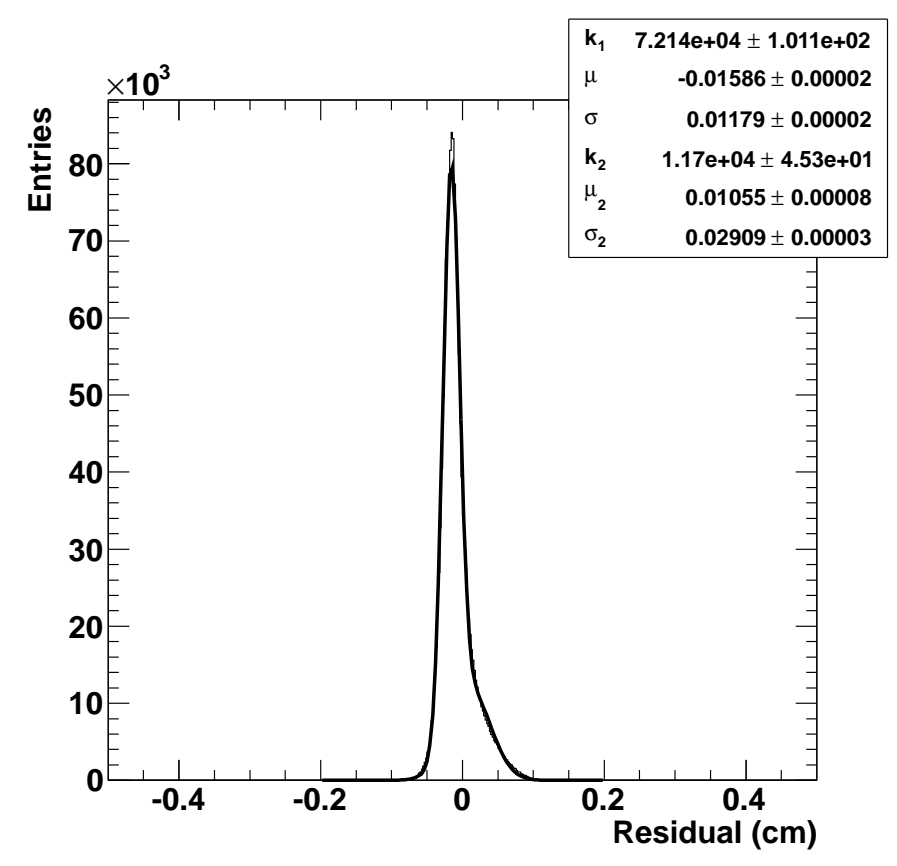

Figure 12. Residual distribution obtained with ${ }^{12} \mathrm{C} 80 \mathrm{MeV} / \mathrm{u}$ beam, operating the Beam Monitor at $1.8 \mathrm{kV}$ with an $\mathrm{Ar} / \mathrm{CO}_{2} 80 \% / 20 \%$ gas mixture. A double Gaussian fit is superimposed.

gas mixture of $\mathrm{Ar} / \mathrm{CO}_{2} 80 \% / 20 \%$ is shown in figure 12. Spatial resolution results are shown as a function of gas mixtures and high voltages in figure 13. Test performed with the highly ionizing ${ }^{12} \mathrm{C} 80 \mathrm{MeV} / \mathrm{u}$ beam confirmed that a space resolution of $\sim 60 \mu \mathrm{m}$ can be achieved.

The study of residuals has been carried out also as a function of the track distance from the sense wire, building the residual distributions in several ranges of track-wire distances. The results are shown for electrons (BTF) and protons (LNS) in figure 14 left and right respectively. 


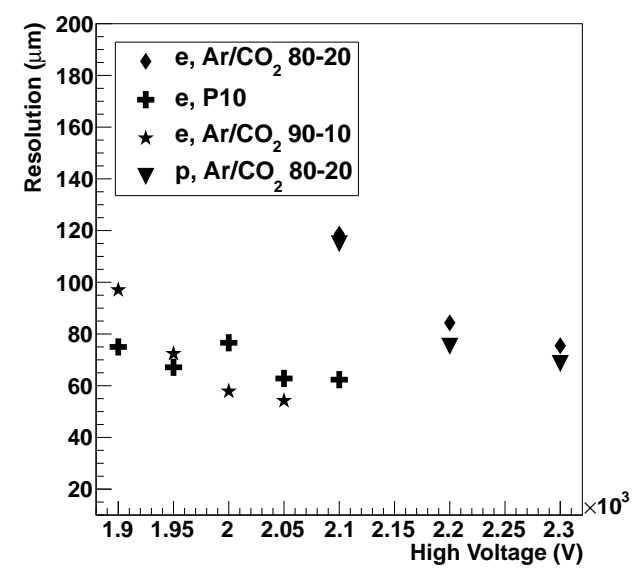

Figure 13. Beam Monitor spatial resolution $(\sigma)$ obtained for electrons (BTF data) and protons (LNS data) shown as a function of the applied voltage for different gas mixtures.
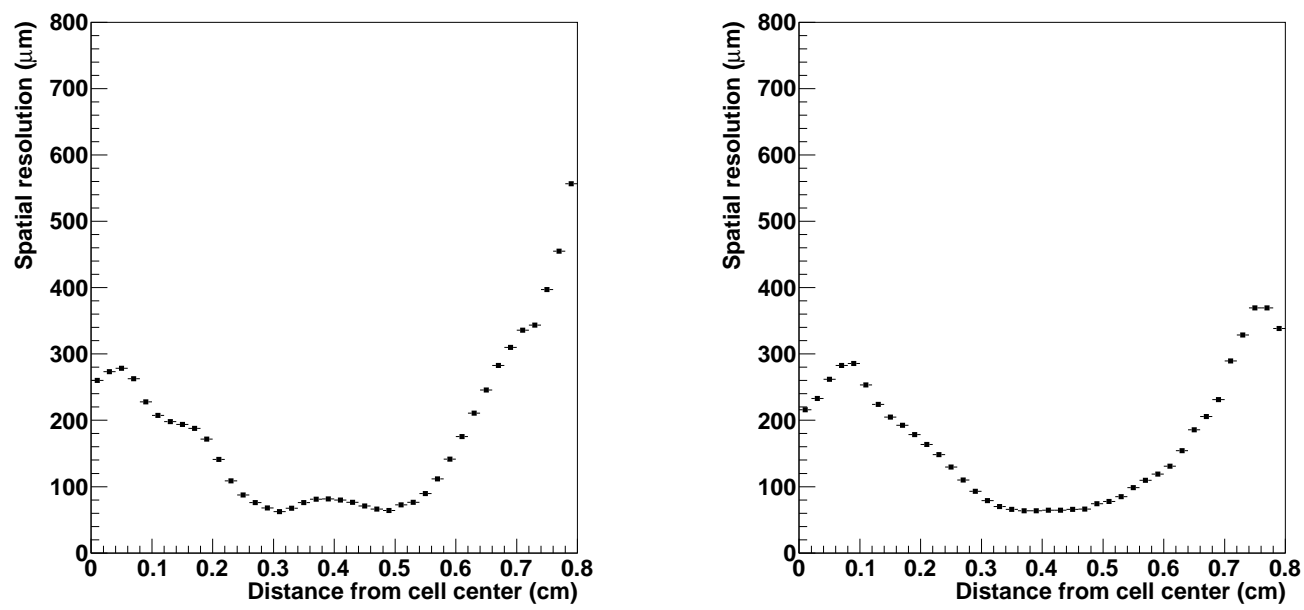

Figure 14. Beam Monitor single cell spatial resolution $(\sigma)$ as a function of the distance from the sense wire. Left plot refer to electrons (BTF), right one to protons (LNS).

\section{Detector performance in the FIRST experiment at GSI}

The FIRST experiment took data on August 2011, performing $400 \mathrm{MeV} / \mathrm{u}{ }^{12} \mathrm{C}$ collisions on $8 \mathrm{~mm}$ graphite and Au targets. The Interaction Region installation at GSI is shown in figure 15.

\subsection{Start Counter preliminary results}

The Start Counter performances have been measured with data from $400 \mathrm{MeV} / \mathrm{u}{ }^{12} \mathrm{C}$ ions. In order to asses the efficiency, two different strategies have been used: the first requires at least one photomultiplier fired when a carbon ion is traversing the detector, while the second strategy requires, instead, that all the photomultipliers must be fired. The efficiency, for an applied threshold of $30 \mathrm{mV}$, showed an excellent stability during the data taking: a mean value of $99.7 \%$ with and 


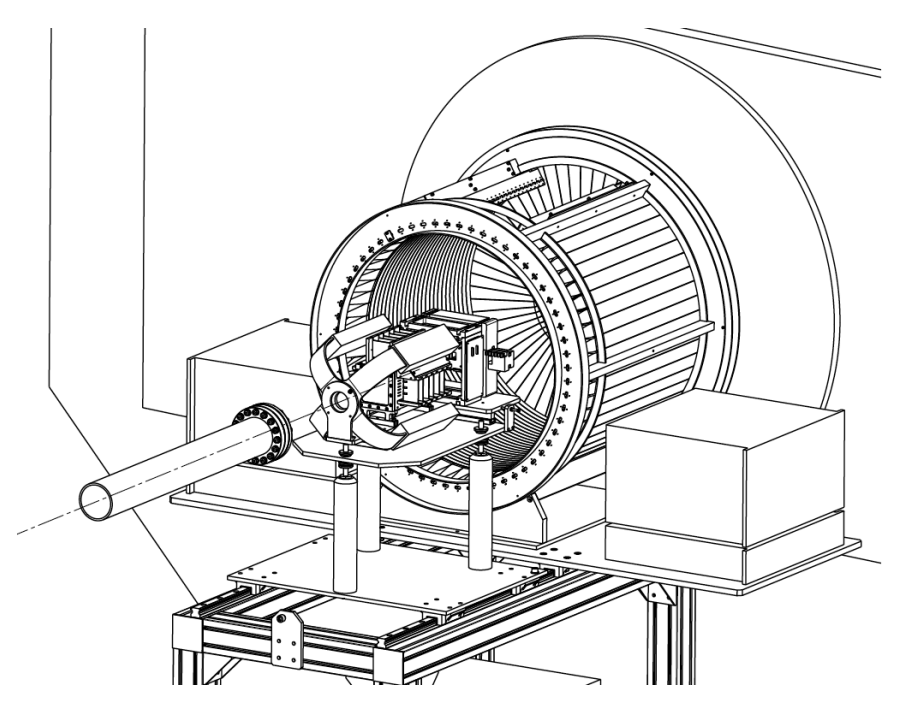

Figure 15. Interaction Region installation at GSI.

RMS of $0.15 \%$ is measured using both strategies. Since the results agree within the statistical uncertainties one can infer that whenever a photomultiplier is fired all the others are fired as well. The efficiency has been evaluated with respect to a trigger signal only for a subset of the collected data.

Also the time resolution has been measured as a function of the run number by fitting the time difference distribution between two selected photomultipliers with a Gaussian function. The time resolution showed an outstanding stability (RMS $\sim 2 \mathrm{ps}$ ) during all the experiment, with a maximum fluctuation of $\approx 5 \mathrm{ps}$ around an average value of $\sigma_{t} \approx 150 \mathrm{ps}$ (that is, as expected, a behavior comprised between that of protons and of $80 \mathrm{MeV} / \mathrm{u}$ carbon ions used during our tests).

Those small fluctuations are also an indication of the very good performance uniformity of the Start Counter over its area, since during the various runs the beam spot was moved in the horizontal (x) and vertical (y) directions for several millimiters (see section 5.2 and figure 19).

\subsection{Beam Monitor preliminary results}

The Beam Monitor was operated, at GSI, at $1.8 \mathrm{kV}$ with an $\mathrm{Ar} / \mathrm{CO}_{2}(80 \% / 20 \%)$ gas mixture. A typical example of particle reconstructed trajectory (from a carbon ion traversing the detector), is shown in figure 16. The size of the circles is directly proportional to the particle distance from the wire.

The number of hits detected with the drift chamber when the detector is perfectly perpendicular to the beam, is 12 (six on each view). With the FIRST data we observe a distribution centered around 13 with and RMS of $\sim 0.3$. That is a proof of the very good stability of the Beam Monitor performances during the data taking and an indication of a slight misalignment of the Beam Monitor with respect to the beam direction, causing a geometrical cross-talk effect.

Drift chamber spatial resolution, evaluated using FIRST data of ${ }^{12} \mathrm{C}-\mathrm{C}$ collisions by means of the same strategy discussed in section 4.3 , has an average value of $\sigma_{x}=140 \mu \mathrm{m}$. Once the residuals distribution is obtained, an additional study has been carried out in order to measure the spatial resolution as a function of the wire distance. The results of this dedicated study are reported for a selected run in figure 17 . 


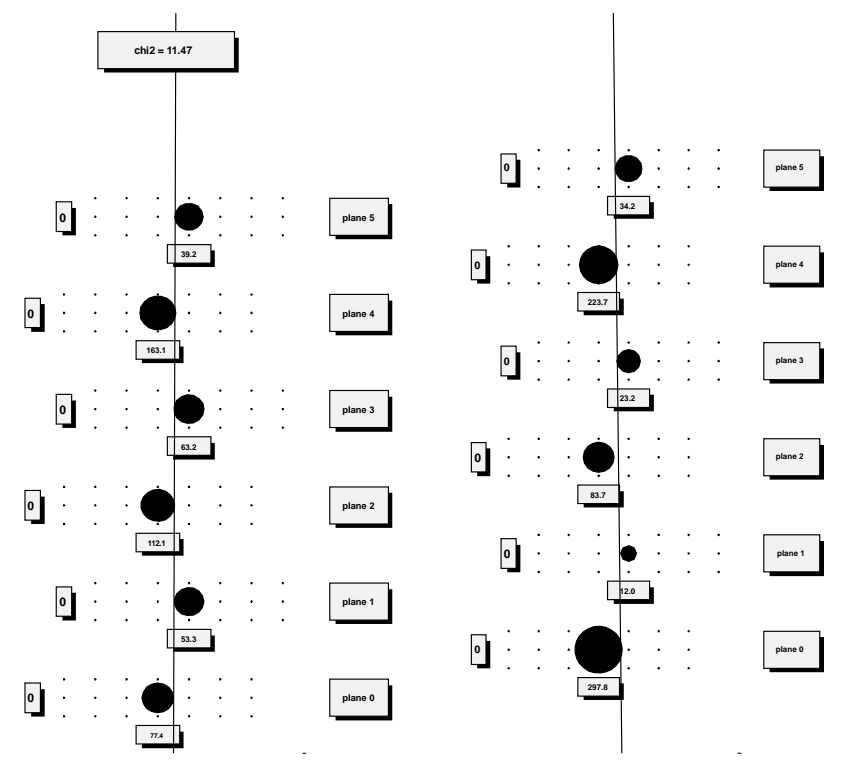

Figure 16. Beam Monitor event display for carbon ions traversing the detector.

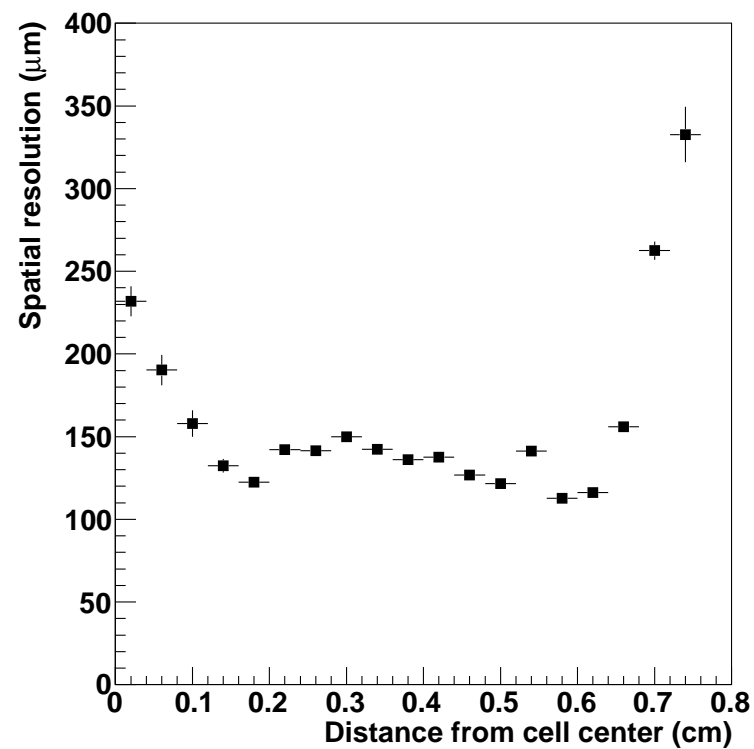

Figure 17. Beam Monitor spatial resolution as a function of the distance from the cell center (sense wire). 


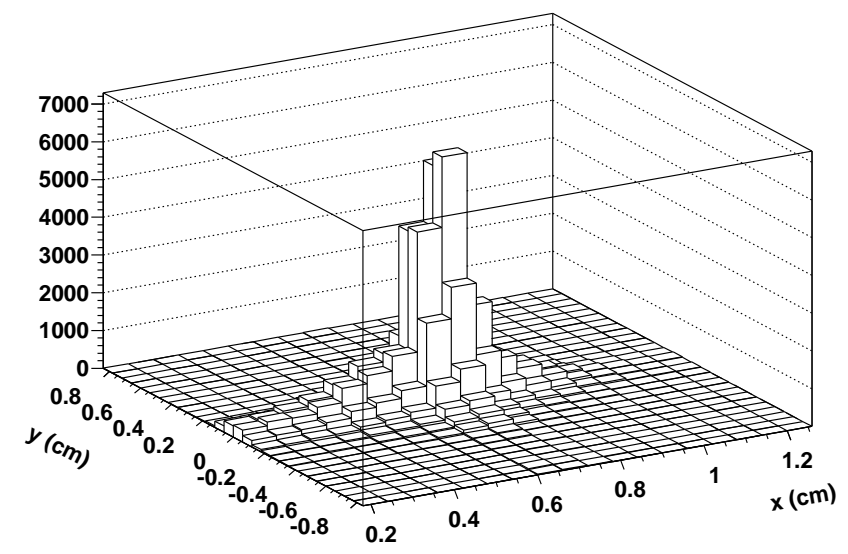

Figure 18. GSI beam spot as reconstructed by the drift chamber.



Figure 19. Mean beam spot position (BS), in the $x, y$ plane, recostructed using the Beam Monitor from the full FIRST data sample. Each point is related to a given run.

Finally, after all the tracks have been reconstructed, we could measure the GSI beam spot as it was seen by the drift chamber just before the target, shown in figure 18. The mean beam spot position, as reconstructed in the various runs taken from FIRST experiment is shown in figure 19, showing that variations of few millimiters occurred during the data taking.

\section{Conclusions}

We have developed the upstream Interaction Region detectors for the FIRST experiment: the Start Counter, a fast scintillator ( $250 \mu \mathrm{m}$ thick) used for trigger and timing purposes and the Beam Monitor, a drift chamber used for particle trajectories tracking. 
A functional test of FIRST upstream Interaction Region detectors has been carried out at the LNF Beam Test Facility and at the LNS Zero Degree Line. The aim of the test was to verify the Beam Monitor and Start Counter performances as well as to find the most suitable working point in GSI-like conditions. Both detectors have been successfully operated, fulfilling FIRST requirements: a Start Counter time resolution less than $250 \mathrm{ps}$ and a Beam Monitor spatial resolution of the order of $100 \mu \mathrm{m}$ have been measured in a wide range of configurations. The FIRST experiment took data on August 2011, performing $400 \mathrm{MeV} / \mathrm{u}{ }^{12} \mathrm{C}$ on $8 \mathrm{~mm}$ graphite collisions ( $\approx 18 \mathrm{M}$ events) and ${ }^{12} \mathrm{C}$ on $\mathrm{Au}(\approx 2 \mathrm{M}$ events). Both the detectors ran smoothly, showing a very stable behavior for the entire duration of the experiment. The detectors have completely matched the expectations: the measured time and spatial resolution are respectively $\sigma_{t} \approx 150 \mathrm{ps}$ and $\sigma_{x} \approx 140 \mu \mathrm{m}$.

\section{Acknowledgments}

We would like to thank B. Buonomo L. Foggetta, A. Mengucci and F. Romano for their generous help in the preparation and pursuance of the BTF and LNS test. We would like to acknowledge M. Arba, L. La Delfa and M. Tuveri (INFN Sez. Cagliari), M. Anelli, S. Cerioni, G. Corradi, D. Riondino and R. Rosellini (INFN, LNF), M. Magi (Dipartimento di Scienze di Base e Applicate per l'Ingegneria, Università di Roma "La Sapienza"), M. Capponi and A. Iaciofano (INFN, Sez. Roma3) for the technical design and mechanical work on the Interagion Region, and Filippo Bosi (INFN Sez. Pisa) for his help and suggestions. We would like also to acknowledge the support given to the FIRST experiment from Prof. C. Scheidenberger and the GSI accelerator staff. This work has been supported by the European Community FP7 - Capacities, contract ENSAR ${ }^{\circ}$ 262010. This work was also supported by Junta de Andalucia and the Spanish Ministerio de Ciencia e Innovacion Contracts P07-FQM-02894, FIS2008-04189 and FPA2008-04972-C03. Finally some of the authors would like to thank CNRS-In2p3 for the support. The research leading to these results has received the financial support of the Belgian company Ion Beam Applications (IBA).

\section{References}

[1] V. Patera, Nuclear physics experiment for hadrontherapy application, Nuovo Cim. C 34 (2011) 179.

[2] J. Hubele et al., Fragmentation of gold projectiles: From evaporation to total disassembly, Z. Phys. A 340 (1991) 263.

[3] G. Bauer et al., A multiple sampling time projection ionization chamber for nuclear fragment tracking and charge measurement, Nucl. Instrum. Meth. A 386 (1997) 249.

[4] LAND collaboration, T. Blaich et al., A Large area detector for high-energy neutrons, Nucl. Instrum. Meth. A 314 (1992) 136.

[5] P. Avery, Applied Fitting Theory I, General Least Squares Theory, (1991), http://phys.ufi.edu/avery/fitting/fitting1.pdf. 